\title{
ЦЕЛЕВАЯ ФУНКЦИЯ ТОЧНОСТИ ОПРЕДЕЛЕНИЯ ГЕОДАННЫХ
}

\author{
А. В. Воронин \\ Федеральный исследовательский иентр «Информатика и управление» Российской академии наук
}

\section{Поступила в редакцию 11.12.2018 г.}

\begin{abstract}
Аннотация. Статья продолжает тематический цикл статей, посвященных геоинформационным системам. Рассмотрена функция точности определения геопространственных данных, формирующих оверлейный слой в геоинформационной системе (ГИС). Целевая функция описывает рассогласование между значениями координат истинного местоположения геообъекта и полученными в процессе вычисления. Приведены выражения и получены графики зависимости точности определения координат геообъектов от значений пространственной и временной характеристик.

Ключевые слова: целевая функция, точность, геоинформационная система, ГИС, геообъект, геоданные.

Annotation. A function of precise geospatial data identification, forming an overlay layer in the geoinformation system has been considered. The target function describes a mismatch between coordinate values of real geoobject's position and values received in the computation process. The expressions are given and graphs of the accuracy of determining the coordinates of geo objects on the values of the spatial and temporal characteristics are obtained.

Keywords: target function, precision, geoinformation system, GIS, geoobject, geodata.
\end{abstract}

\section{ВВЕДЕНИЕ}

Принятый Правительством Российской Федерации курс по реализации программы цифровой экономики (ЦЭ) находится с одной стороны в полном соответствии с тенденциями развития экономических процессов в мире [1], с другой требует развития в России основных инфраструктурных элементов ЦЭ, для реализации которых определены семнадцать федеральных проектов $[2,3]$. С точки зрения информатики и управления особый интерес представляют: «Информационная инфраструктура» (Министерство цифрового развития, связи и массовых коммуникаций Российской Федерации, ПАО «Ростелеком», АНО «Цифровая экономика»), «Информационная безопасность» (Министерство цифрового развития, связи и массовых коммуникаций Российской Федерации, ФСТЭК России, ПАО «Сбербанк», АНО «ЦКИКТ», АНО «Цифровая экономика»), «Цифровые технологии» (Министерство цифрового развития, связи и массовых коммуникаций Рос-

(c) Воронин А. В., 2019 сийской Федерации, Министерство высшего образования и науки Российской Федерации, ГК «Росатом», ГК «Ростех», АНО «Цифровая экономика»).

Для успешной реализации проектов требуется системный подход к формированию базовых направлений и последующих положений, суть которых заключается в определении основных организационных, методологических и системотехнических решений, а именно: системы целевых показателей по этапам развития, состава функциональных задач, организации по уровням и иерархии структурного построения, принципов информационного взаимодействия, совместного функционирования, мониторинга и обеспечения информационной безопасности [4-6]. Решения в рамках этого системного направления являются задающими для всех остальных направлений и проектов ЦЭ.

Развитие же инфокоммуникационного мира [7-9] требует активного использования вычислительных средств, а так же систем управления и принятия решений. Данные системы, являясь составной частью информационной среды, обрабатывают большие объе- 
мы разнородных данных, формируя массивы знаний для принятия решений в различных отраслях деятельности [10]. Такие системы со средствами визуализации и учетом географического местоположения разнородных данных образуют геоинформационные системы (ГИС) $[11,12]$. Отечественные Hi-Tech-производители активно осваивают рынок разработки и сопровождения ГИС [13]. Однако в случае отображения специализированных геоданных (объекты недвижимости, связи, управления) вначале требуется определение координат (местоположения) этих геообъектов. Точность расчета координат геообъектов специализированного (оверлейного) слоя позволяет точно отобразить их в геоинформационной системе.

Целью исследования является определение целевой функции рассогласования (точности) между значением координаты, полученной в процессе расчета местоположения специализированного геообъекта, и значением истинной координаты его базирования.

Целевая функция позволит рассчитывать точность представления геопространственных данных (геообъектов) оверлейного слоя в геоинформационной системе.

\section{1. ЦЕЛЕВАЯ ФУНКЦИЯ ТОЧНОСТИ ОПРЕДЕЛЕНИЯ ГЕОПРОСТРАНСТВЕННЫХ ДАННЫХ}

Согласно $[14,15]$ известно, что координаты пространственного объекта, как правило, являются зависимыми величинами (например, в геоцентрической системе $(x, y, z))$. Анализ точности положения таких объектов выполняется на основе строгой оценки в соответствии со способом математической обработки результатов. Оценка точности осуществляется с использованием тензора ошибок. Тензор является идеальным инструментом для анализа геопространственных данных независимым от выбора системы координат, однако не позволяет учесть влияние параметров характеристик, используемых при расчете определения местоположения геообъекта.

В качестве целевой функции, характеризующей точность определения положения гео- пространственных объектов, целесообразно использовать рассогласование значения координат, полученных в процессе вычислений при решении задачи определения местоположения объекта оверлейного слоя ГИС, от истинной координаты его базирования. Рассогласование координат целесообразно определять через разность векторов $\vec{k}_{p} ; \vec{k}_{u}\left(\vec{k}_{p}-\right.$ вектор рассчитанных координат; $\vec{k}_{u}-$ вектор истинных координат) с центрами в геоцентрической системе и концами в расчетной и истинной точках местоположения объекта:

$$
\vec{p}=\vec{k}_{p}-\vec{k}_{u},
$$

где $\vec{k}_{p}$ - вектор рассчитанных координат, полученных в процессе вычисления при решении задачи определения местоположения геообъекта;

$\vec{k}_{u}$ - вектор истинных координат базирования геообъекта.

Величина рассогласования рассчитывается как длина вектора рассогласования

$$
|\vec{p}|=\sqrt{p_{x}^{2}+p_{y}^{2}+p_{z}^{2}},
$$

где $p_{x}, p_{y}, p_{z}$ - координаты вектора рассогласования, определяемые как

$$
\begin{aligned}
& p_{x}=k_{p_{x}}-k_{u_{x}} \\
& p_{y}=k_{p_{y}}-k_{u_{y}}, \\
& p_{z}=k_{p_{z}}-k_{u_{z}}
\end{aligned}
$$

где $k_{p_{x}}, k_{p_{y}}, k_{p_{z}}$ - координаты вектора рассчитанных координат;

$k_{u_{x}}, k_{u_{v}}, k_{u_{z}}-$ координаты вектора истинных координат.

Алгоритмы расчета пространственного положения объектов, как правило, сопряжены с использованием значений параметров временных характеристик (например, [16, 17]). В свою очередь пространство (расстояние $-l$ ) и время $(t)$ связаны через скорость света выражением:

$$
l=c t,
$$

где $c$ - скорость света, равная $3 \times 10^{8} \mathrm{M} / \mathrm{c}$.

Следовательно, логична запись целевой функции, характеризующей точность определения геопространственных данных в следующем виде 


\section{А. В. Воронин}

$$
\begin{gathered}
p(t)= \\
=\sqrt{\left(k_{p_{x}}(t)-k_{u_{x}}\right)^{2}+\left(k_{p_{y}}(t)-k_{u_{y}}\right)^{2}+\left(k_{p_{z}}(t)-k_{u_{z}}\right)^{2}} .
\end{gathered}
$$

В результате в качестве целевой функции, характеризующей точность определения геопространственных данных (5), целесообразно использовать рассогласование значений координат, полученных в процессе определения (вычисления) местоположения геообъекта, от истинной координаты его базирования. Учитывая (4), точность вычисления местоположения геообъекта также определяется значением временной характеристики, участвующей в расчете координаты объекта.

\section{2. РЕЗУЛЬТАТЫ ИССЛЕДОВАНИЯ ЦЕЛЕВОЙ ФУНКЦИИ ТОЧНОСТИ ОПРЕДЕЛЕНИЯ ГЕОПРОСТРАНСТВЕННЫХ ДАННЫХ}

Исследование целевой функции точности определения геоданных осуществлялось при различных значениях пространственной и временной характеристик. Пределы простран- ственной характеристики составили от 0 до 3 000 м, временной - от 0 до 10 мкс. Выбор значений пределов обусловлен наиболее часто встречаемой погрешностью измерений параметров пространственной и временной характеристик, полученных в процессе вычислений при решении задачи определения местоположения геообъекта. Зависимость изменения значений пространственной характеристики $(l)$ от временной $(t)$ представлена на рис. 1.

График (рис. 1) представляет монотонно возрастающую линейную зависимость. В абсолютных значениях изменение значения пространственной характеристики значительно, так при значении временной характеристики 1 мкс изменение пространственной составляет 300 м. При значении временной характеристики 10 мкс изменение пространственной $-3000 \mathrm{~m}$.

График зависимости изменения относительных значений пространственной характеристики от значений временной представлен на рис. 2.

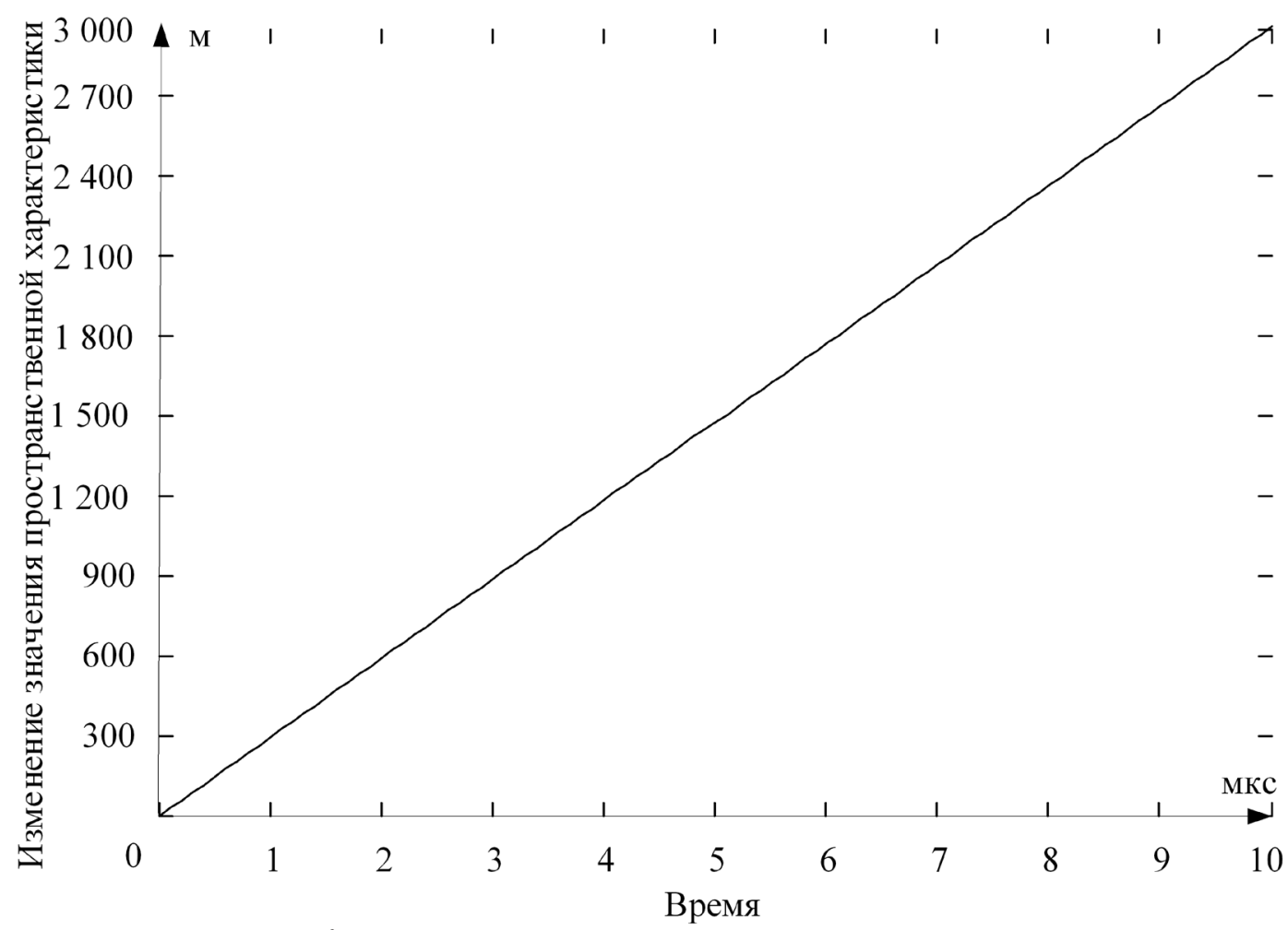

Рис. 1. График зависимости изменения значений пространственной характеристики от временной 
Целевая функция точности определения геоданных ...

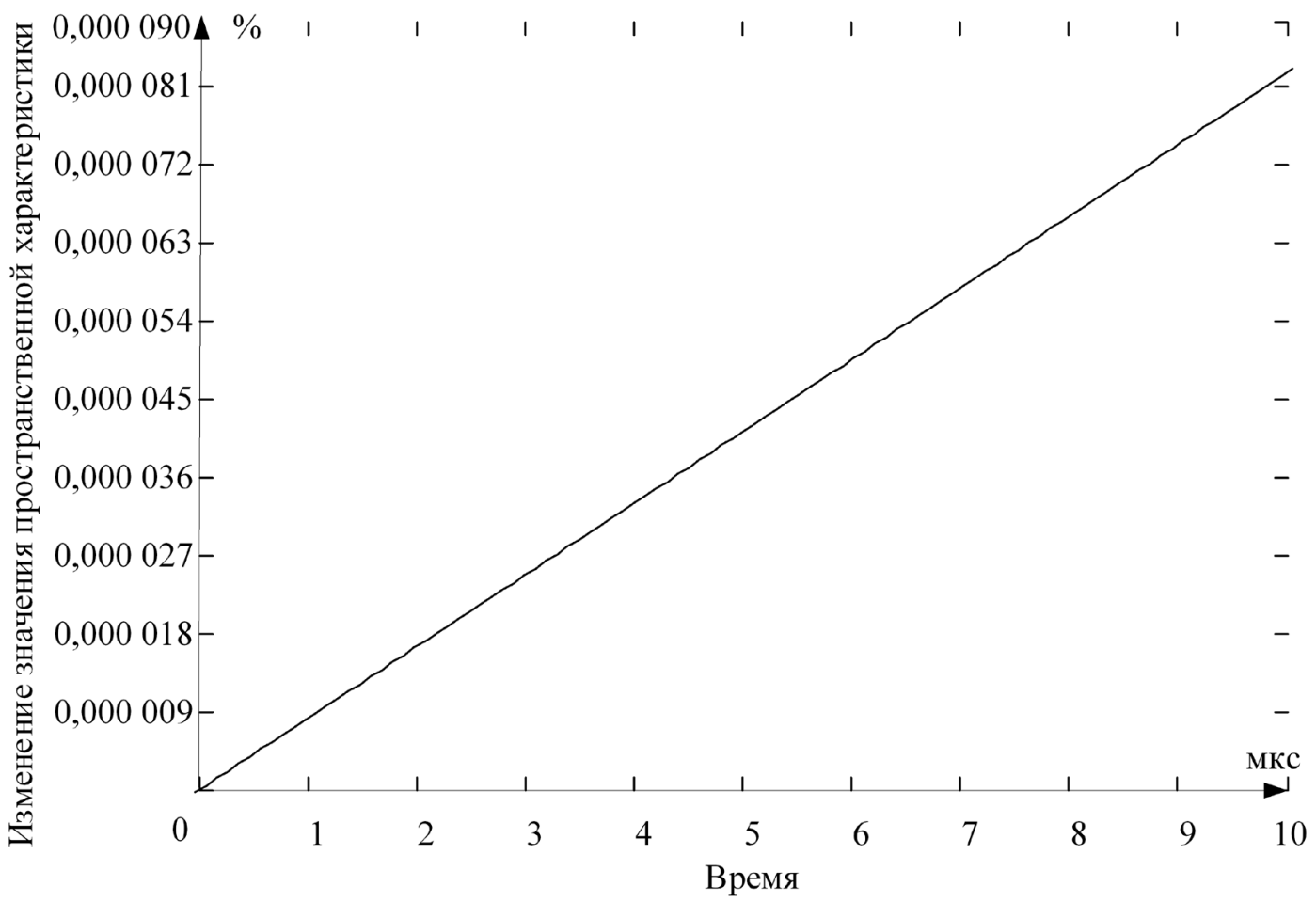

Рис. 2. График зависимости изменения относительных значений пространственной характеристики от значений временной

Таблица 1

Точность определения координат геообъекта

\begin{tabular}{|l|c|c|c|c|c|c|c|c|c|c|}
\hline & & & & & & & & & & \\
\hline $\begin{array}{l}\text { Значение временной } \\
\text { характеристики (мкс) }\end{array}$ & 0,5 & 1 & 1,5 & 2 & 2,5 & 3 & 3,5 & 4 & 4,5 & 5 \\
\hline $\begin{array}{l}\text { Значение } \\
\begin{array}{l}\text { пространственной } \\
\text { характеристики (км) }\end{array}\end{array}$ & 0,15 & 0,3 & 0,45 & 0,6 & 0,75 & 0,9 & 1,05 & 1,2 & 1,35 & 1,5 \\
\hline $\begin{array}{l}\text { Точность определения } \\
\text { координат (км) }\end{array}$ & 1 & 2,5 & 3 & 5 & 7,5 & 10 & 14,5 & 19 & 24,5 & 32,5 \\
\hline
\end{tabular}

Нормировка изменения пространственной характеристики $\left(O_{l}\right)$ приведена к минимальному значению пространственной характеристики (в процентах):

$$
O_{l}=\frac{c t}{L_{\min }} \cdot 100
$$

где $L_{\min }$ - минимальное значение пространственной характеристики.

Точность определения координат геообъекта является расстоянием между реальными координатами геообъекта и координатами, полученными в процессе вычисления (табл. 1, рис. 3).

График зависимости точности определения координат геообъекта от значений про- странственной характеристики (значений временной характеристики) имеет возрастающий характер. Как видно из таблицы и зависимости на графике, точность определения координат геообъекта с увеличением значения временной характеристики, приводящей к увеличению значения пространственной характеристики, ухудшается. Так при значении временной характеристики 0,5 мкс, соответствующей изменению значения пространственной характеристики 0,15 км, точность определения координат геообъекта составляет 1 км. При значении временной характеристики 5 мкс (изменение значения пространственной характеристики 1,5 км), 


\section{А. В. Воронин}

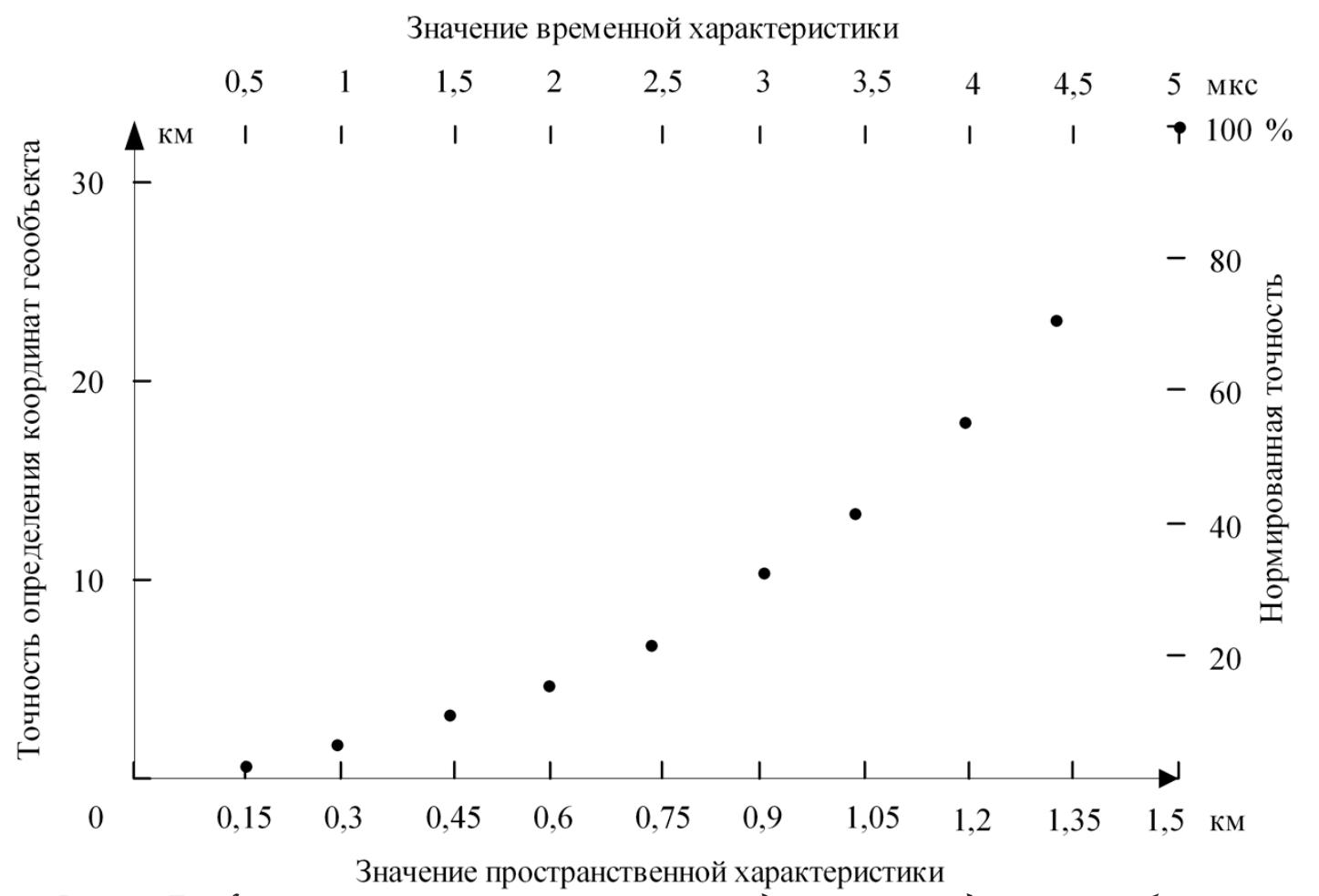

Рис. 3. Грабик зависимости точности определения координат геообъекта от значений пространственной характеристики (временной характеристики)

точность определения координат геообъекта равна 32,5 км.

Таким образом, для успешной реализации проектов ЦЭ требуется системный подход к формированию базовых направлений и показателей их оценки. Развитие же инфокоммуникационного мира, как базового элемента цифровой экономики, требует активного использования вычислительных средств, а так же систем управления и принятия решений. Данные системы, являясь составной частью информационной среды, обрабатывают большие объемы разнородных данных, создавая массивы знаний для принятия решений в различных отраслях деятельности, формируя со средствами визуализации геоинформационные системы, оценить которые возможно через точность расчета координат геообъектов оверлейного слоя ГИС, отображаемых в геоинформационной системе. По результатам исследования не только предложена, но и исследована функция точности отображения геопространственных данных, формирующих специализированный слой в геоинформационной системе. Функция характеризует рассогласование между значениями коорди- нат истинного местоположения геообъекта и полученными в процессе вычисления. График зависимости точности определения координат геообъекта от значений пространственной характеристики (значений временной характеристики) имеет возрастающий характер, то есть точность определения координат геообъекта с увеличением значения временной характеристики, приводящей к увеличению значения пространственной характеристики, ухудшается.

\section{ЗАКЛЮЧЕНИЕ}

Развитие цифровой экономики затрагивает практически все аспекты человеческой деятельности и оказывает влияние на каждого человека независимо от его профессии, рода занятий, возраста и социального положения. ЦЭ предполагает решение задач по созданию единого информационного реестра всех ресурсов в цифровой экономике, созданию и внедрению технологии учета всех процессов по изменению этих ресурсов и обеспечению наполнения (обновления) единого реестра ресурсов актуальными, достоверными и объ- 
ективными исходными данными. Масштабность, сложность, многоплановость и междисциплинарность задач, решаемых в рамках программы цифровой экономики, вызывает объективную необходимость по определению целевых показателей оценки развиваемых направлений. Одним из направлений развития ЦЭ является инфокоммуникационная сфера, требующая активного использования вычислительных средств, а так же систем управления и принятия решений. Данные системы, являясь составной частью информационной среды, формируют со средствами визуализации геоинформационные системы, одна из оценок которых заключается в точности представления геообъектов в оверлейном слое ГИС. Исходя из анализа предметной области, предложен показатель точности отображения геопространственных данных, формирующих специализированный слой в геоинформационной системе. Исследование поведения показателя осуществлено через анализ целевой функции, характеризующей рассогласование между значениями координат истинного местоположения геообъекта и полученными в процессе вычисления при различных значениях пространственной и временной характеристик (пределы пространственной характеристики: 0 и 3000 м; временной: 0 и 10 мкс). Получены выражения и графики зависимости точности определения координат геообъектов от значений пространственной и временной характеристик.

\section{СПИСОК ЛИТЕРАТУРЫ}

1. Шваб, К. Четвертая промышленная революция / К. Шваб. - Москва : Эксмо, 2016. 208 c.

2. О Стратегии развития информационного общества в Российской Федерации на 2017-2030 годы : указ Президента Российской Федерации от 09 мая 2017 г. № 203. - Режим доступа: http://www.kremlin.ru/acts/bank/41919

3. Программа «Цифровая экономика Российской Федерации» : распоряжение Правительства РФ от 28.07.2017 г. №1632-р. - Режим доступа: http://static.government.ru/media/files /9gFM4FHj4PsB79I5v7yLVuPgu4bvR7M0.pdf
4. Зацаринный, А. А. Информационные технологии в цифровой экономике / А. А. Зацаринный // Проектирование будущего. Проблемы цифровой реальности : труды 1-й Международной конференции (Москва, 8-9 февраля 2018 г.). - Москва, ИПМ им. М. В. Келдыша, 2018. - С. 29-35.

5. Управление научными сервисами как основа национальной цифровой платформы «Наука и образование» / А. К. Горшенин [и др.] // Стратегические приоритеты. 2017. - №2. - С. 103-114.

6. Зацаринный, А. А. Некоторое представление жизненного цикла информационных систем стран Большой Евразии / А. А. Зацаринный, А. В. Воронин // Аналитика развития, безопасности и сотрудничества: Большая Евразия-2030 : мматериалы IV Международной научно-практической конференции (Москва, 29 ноября 2017 г.). - Москва, КогитоЦентр, 2017. - С. 66-70.

7. Marr, D. Vision: A computational investigation into the human representation and processing of visual information. - Massachusetts : MIT press, 2010. - $362 \mathrm{p}$.

8. Zenus, $M$. Is IoT data taking geospatial analytics to the next level? - Geospatial World, 2018, august. $-4 \mathrm{~s}$.

9. Wilson, K. Geospatial data fuel for the digital economy. - Geospatial World, 2018, september. $-4 \mathrm{~s}$.

10. Воронин, А. В. Классификация геообъектов в геоинформационной системе с использованием метаданных и математического аппарата теории нечетких множеств (нейронных сетей) / А. В. Воронин // Математическое моделирование и информационные технологии в инженерных и бизнес-приложениях : сборник материалов международной научной конференции (Воронеж, 3-6 сентября 2018 г.). - Воронеж, Издательский дом ВГУ, 2018. - С. 189-196.

11. Воронин, А. В. Результаты анализа перспектив развития геоинформационных систем / А. В. Воронин // Журнал «Системы высокой доступности». - 2017. - № 4, Т. 133. C. 68-75.

12. Воронин, А. В. Анализ методов и способов функционирования геоинформационных 


\section{А. В. Воронин}

систем / А. В. Воронин // Журнал «Информация и Космос». - 2018. - № 3. - С. 124-131.

13. Ионенков, Ю. С. Особенности оценки эффективности геоинформационной системы как элемента ситуационного центра / Ю. С. Ионенков, А. А. Зацаринный // Журнал «Системы и средства информатики». - 2018. T. 28, № 2. - С. 75-87.

14. Хатоум, Т. С. Критерии оценки точности геопространственных данных / Т. С. Хатоум. - Новосибирск : СГГА, 2007. - 6 с.

15. Коробков С. А. Тензор ошибок на плоскости и в пространстве / С. А. Коробков // Изв. вузов. Геодезия и аэрофотосъемка. 2000. - № 2. - С. 3-19.

16. Способ определения местоположения VSAT-станции в спутниковой сети : пат. 2450284 Российская Федерация : МПК

Воронин Алексей Владимирович - канд. техн. наук, доцент, ведущий научный сотрудник, Федеральный исследовательский центр «Информатика и управление» Российской академии наук.

Тел.: 8(499)135-41-89

E-mail: aleksey.v.v@mail.ru
G01S5/02 / Иванов B. Н. ; заявитель и патентообладатель государственное казенное образовательное учреждение высшего профессионального образования Академия Федеральной службы охраны Российской Федерации. - № 2010121505/07 ; заявл. 26.05.10 ; опубл. 10.05.12, Бюл. № 13. - 11 с.

17. Способ определения местоположения станции сети связи VSAT : пат. 2606958 Российская Федерация : МПК G01S5/02 / Трофименков С. А., Иванов В. Н. ; заявитель и патентообладатель государственное казенное образовательное учреждение высшего профессионального образования Академия Федеральной службы охраны Российской Федерации. - № 2016102880 ; заявл. 28.01.16 ; опубл. 10.01.17, Бюл. № 1. - 14 с.

Voronin Aleksey Vladimirovich - PhD Tech., Associate Professor, Research Scientist, Federal Research Center «Computer Science and Control» of RAS.

Tel.: 8(499)135-41-89

E-mail: aleksey.v.v@mail.ru 